\title{
A DESCRIPTION OF ALL GLOBALLY IDEMPOTENT THREADS WITH ZERO $\left({ }^{1}\right)$
}

\author{
BY \\ CHARLES R. STOREY $\left({ }^{2}\right)$
}

The term "thread" was introduced by A. H. Clifford in [1] to designate a connected topological semigroup in which the topology is that induced by a total order relation. A thread $S$ is said to be globally idempotent if $S^{2}=S$. In [6] the author has shown that, after reversing the order if necessary, the subset $\{x \mid 0 \leqq x\}$ in a globally idempotent thread with zero is a subthread having a particularly pleasant structure. This result is the foundation on which the description given in this paper is based.

An analogous situation existed in the characterization given by Cohen and Wade [4] of all topological semigroups on a compact real interval which have a zero and an identity. In such a thread, the identity must be an endpoint, and the closed interval between the zero and the identity a subthread. Assuming the identity to be the maximal element, this subthread is then, in the terminology of [1], a standard thread. Since a characterization of all standard threads on a real interval had previously been given by Mostert and Shields [5], the problem solved by Cohen and Wade was also that of utilizing a given structure theorem for $\{x \mid 0 \leqq x\}$ to formulate a description of the whole thread. Consequently, many of the ideas developed by Cohen and Wade have again been used here.

Treating the same type of problem, Clifford determined in [1] all possible compact threads having a zero and idempotent endpoints, and again, some of our results are simple generalizations of those in [1]. Considering the work of Clifford and of Cohen and Wade, the contribution of the present investigation toward a description of all globally idempotent threads with zero lies in dropping the requirement of compactness and in replacing the assumption of idempotent endpoints or of an identity by the weaker one of global idempotency.

The terminology and notation will be essentially the same as that used in [6]. In particular, a standard thread is a compact thread in which the least element is a zero and the largest is an identity. We note that the trivial thread consisting of a zero alone is considered a standard thread. A thread with a zero and an identity

Received by the editors July 25,1960 .

(1) This paper was prepared while the author held a National Science Foundation Postdoctoral Fellowship.

$\left({ }^{2}\right)$ This paper was accepted in January, 1961. An illness that ultimately proved fatal prevented the author from making the few modifications suggested by the referee and returning the manuscript to the editor. At the referee's request, and with his help, the manuscript was retrieved and is being published here posthumously. D.Z. (Editor). 
is a positive thread provided the zero is a least element and provided there is no largest element. The structure theorem for standard threads can be found in [1], [4], [5] or [6]; any positive thread is the contact extension (see $\S 1$ ) of a standard thread by the thread of nonnegative real numbers under ordinary multiplication. In a thread with a zero, we write $x<y$ if and only if $0 \leqq x<y$ or $y<x \leqq 0$. Finally, and this notation is different from that in [6], if $S$ is a thread with zero, we put $L=\{x \mid x \leqq 0\}$ and $G=\{x \mid 0 \leqq x\}$.

The following two theorems, which will be used without explicit reference, are the principal results of [6]. If $S$ is a globally idempotent thread having a zero as a least element, then $S$ is a positive thread, or a standard thread, or the result of removing the identity of a standard thread [6, Theorem 5.5]. If $S$ is a globally idempotent thread with a cutting zero, then either $G^{2}=G$ or $L^{2}=L$; consequently, either $G$ or the order dual of $L$ is described by the preceding statement; moreover, the multiplication in $S$ is monotone with respect to $\prec$, and the zero does not cut $L^{2}, G^{2}, L G$ or $G L[6$, Theorem 6.7].

1. Extensions. We shall say that a thread $S$ is the contact extension of $T$ by $Q$ if and only if $T$ and $Q$ are threads satisfying the hypotheses of 1.1 and $S$ is isomorphic with the thread constructed there. The adjective "contact" was used by Clifford in [1] for a similar extension, however, 1.1 and its converse, 1.2, more closely resemble Lemma 6.1 in [4].

1.1 THEOREM. Let $Q$ be a globally idempotent thread with a zero, let $T$ be a compact globally idempotent thread with zero, say $T=[d, e]$, and define $j: Q \backslash\{0\} \rightarrow T$ by

$$
\begin{aligned}
j(x) & =e, & & \text { if } 0<x, \\
& =d, & & \text { if } x<0 .
\end{aligned}
$$

If $j(x y)=j(x) j(y)$ whenever $x, y$ and $x y$ all lie in $Q \backslash\{0\}$, then the following construction yields a globally idempotent thread $S$ containing $T$ as an ideal such that $S / T=Q$.

Let $S=T \cup Q \backslash\{0\}$ and, thinking of $S$ as obtained by replacing the zero of $Q$ by the interval $[d, e]$, order $S$ in the obvious way. For $x, y$ in $Q \backslash\{0\}$ and for $s, t$ in $T$, define:

$$
\begin{array}{rlrl}
x \circ y & =x y, & & \text { if } x y \neq 0, \\
& =j(x) j(y), & & \text { if } x y=0 ; \\
x \circ s & =j(x) s ; & & \\
s \circ x & =s j(x) ; & & \\
s \circ t & =s t . &
\end{array}
$$

Proof. The hypothesis on $j$ means that $j$ is, in the sense of [3], a ramified homomorphism; and thus by Theorem 2 of that paper, $\circ$ is an associative operation on $S$. The continuity of $\circ$ can be proved by the easy but uninteresting method of considering many cases separately. We note only that the fact that, in $Q$, zero does not cut $L^{2}, G^{2}, L G$ or $G L$ is crucial to the proof. The remaining conclusions are obvious. 
1.2 TheOREM. Let $S$ be a globally idempotent thread with zero and let $T=[d, e]$ be an ideal of $S$. If $G L \subset L$ in $S$, if $e$ is a left identity on $T$, and if either $0<d e$ or $e$ is a right identity on $T$; then $S$ is the contact extension of $T$ by $S / T$.

Proof. Putting $Q=S / T$, it is clear that $T$ and $Q$ are globally idempotent threads with zero. Moreover, we will consider $Q \backslash\{0\}$ as a subset of $S$, so that $S=Q \mid\{0\} \cup T$.

Let $y<d$ and $e<x$. Then by monotony $e=e^{2} \preceq e x, e \preceq x e$, and $d=e d \preceq e y$, while $e x, x e$ and $e y$ are all in $T$. If $0<e$, then $e \preceq e x$ and $e \preceq x e$ imply that $e \leqq e x$ and $e \leqq x e$. Consequently, whether $e$ is zero or not, $e x=x e=e$. Since $G L \subset L, d \preceq e y$ implies $e y \leqq d$, and thus $e y=d$. We have shown that $j(z)=e z$, where $j$ is the function from $Q \mid\{0\}$ into $T$ defined in 1.1, and that $e=x e$ when $e<x$.

Now let $x \in Q \mid\{0\}$ and let $s \in T$. We intend to show that $x s=j(x) s$ and that $s x=s j(x)$. The first of these is trivial, for $x s \in T$ implies $x s=e(x s)=(e x) s=j(x) s$. If $0 \leqq s$ then $s e=s$ and $s x=(s e) x=s(e x)=s j(x)$. Moreover, if $e$ is an identity on $T$, then $s e=s$ for each $s$ and we are through.

Assume that $s \leqq 0$ and that $0<d e$. This means in particular that $L G \subset G$ in $S$. If $e<x$, then $s x \in G \cap T$ and $s x=(s x) e=s(x e)=s e=s j(x)$. Thus we have left only the case where both $s$ and $x$ are in $L$. Since $L G \cup G^{2} \subset G$ while $S$ is globally idempotent, either $L^{2}=L$ or $G L=L$. In the first case the structure of $L$ is known and there exists a $y$ such that $y \leqq d$ and $y^{2}=d$. Thus $d=e d=e y^{2}=(e y) y=d y$ and it follows that $d=(d y) y=d y^{2}=d^{2}$. Now $x<d \leqq s \leqq 0$ implies $s x=s=s d=s j(x)$. On the other hand, if $L=G L$ then there exist an $l$ in $L$ and an $r$ in $G$ such that $r l=x$. Since $x$ is not in $T, l<d$ and $e<r$. Thus $s x=s(r l)=(s r) l=(s e) l=s(e l)=s d=s j(x)$. Hence $x s=j(x) s$ and $s x=s j(x)$ whenever $s \in T$ and $x \notin T$.

If $x, y$ and $x y$ are in $Q \backslash\{x\}$, we have $j(x y)=e(x y)=(e x) y=j(x) y=j(x) j(y)$ because $j(x)$ is in $T$ and $y$ is not. This proves that $j$ is a ramified homomorphism. Finally, if $x$ and $y$ belong to $Q \backslash\{0\}$ and if $x y=0$ in $Q$; then $x y$ belongs to $T$ in $S$, so that $x y=e(x y)=j(x) y=j(x) j(y)$. This concludes the proof.

Before we introduce the next type of extension, we must consider the homomorphisms of a positive thread. In Lemma 8 of [1], Clifford shows that any homomorphism of one standard thread onto another is continuous and order preserving. This is not true for positive threads; for example, define $\phi$ from the positive thread of nonnegative real numbers under ordinary multiplication onto itself by $\phi(x)=x^{-1}$ for $0<x$ and $\phi(0)=0$. However, we do have the following.

1.3 Lemma. Let $S$ be either a standard thread or a positive thread, and let $\phi$ be a continuous homomorphism of $S$ into a standard or positive thread T. Then $\phi$ is order preserving.

Proof. Let $u$ and $e$ be the identities of $S$ and $T$ respectively. If $x \leqq u$, then $x^{n} \rightarrow g$ for some idempotent $g$ in $S$; and since $\phi$ is continuous, $\phi(x)^{n} \rightarrow \phi(g)$. It follows that $\phi(x) \leqq e$, and thus $\phi([0, u]) \subset[0, e]$. If $z<y$ in $S$, there exists an $x$ in $[0, u]$ such that $z=y x$. Then $\phi(z)=\phi(y) \phi(x)$ and $\phi(x) \leqq e$ imply $\phi(z) \leqq \phi(y)$. Hence $\phi$ is order preserving. 
We also have a slight variation of Lemma 9 in [1]. Since the proof is also only a slight variation of the one in [1], we omit it.

1.4 Lemma. Let $S$ be either a standard thread or a positive thread and let $u$ be its identity.

(1) If $\rho$ is a congruence relation with closed convex classes on $S$, then $S / \rho$, ordered in the obvious way, is either a standard thread or a positive thread and the natural homomorphism is continuous. Conversely, if $\rho$ is the congruence relation determined in $S$ by a continuous homomorphism of $S$ into a thread which is also either standard or positive, then $\rho$ has closed and convex classes.

(2) A partition of $S$ into a set of mutually disjoint closed intervals is that of a congruence with closed convex classes if and only if the lower endpoint of each nondegenerate interval in the partition is an idempotent and any nondegenerate interval meeting $H(u)$ contains $H(u) .(H(u)$ is the maximal subgroup of $S$ containing $u$.)

Now let $R$ be a standard thread or a positive thread, let $P$ be the order dual of either a standard or a positive thread, and let $\phi$ be a continuous homomorphism of $R$ into $P$ for which $\phi(0)=0$. Put $\mathscr{N}(P, R, \phi)=P \cup R \mid\{0\}$, identify the zero of $R$ with that of $P$, order $\mathscr{N}(P, R, \phi)$ so that each element of $P$ is less than each nonzero element of $R$, and extend the multiplications given in $P$ and $R$ to $\mathscr{N}(P, R, \phi)$ by $x \circ s=s \circ x=\phi(x) s$ for $x$ in $R$ and $s$ in $P$. Then $\mathscr{N}(P, R, \phi)$ is a right linear extension of $P$ by $R$.

Again, the case in which $R$ and the dual of $P$ are both standard threads was given by Clifford. Lemma 10 of [1] states that $\mathscr{N}(P, R, \phi)$ is a globally idempotent thread with zero in which $L$ and $G$ are isomorphic with $P$ and $R$ respectively, and the proof extends immediately to the more general situation above.

In the construction of an $\mathscr{N}(P, R, \phi), \phi(R)$ is certainly a subthread of $P$. Moreover, by 1.4 the congruence determined in $R$ by $\phi$ has closed convex classes, and $\phi(R)$ is itself the order dual of a standard or positive thread. That is, either $P$ is the dual of a positive thread and $\phi(R)=P$, or there is an idempotent $g$ in $P$ with $\phi(R)=[g, 0]$. (We note that $\phi(R)$ need not be an ideal of $P$, for if $P$ is the dual of a positive thread, $\phi(R)$ might well be the closed interval between the zero and the identity.) Thus, $P$ can be constructed from $R$ by taking a congruence $\rho$ with closed convex classes on $R$, reversing the order in $R / \rho$, and then making a rather simple extension. The special case in which $\phi$ is onto will occur frequently, and we shorten the notation in that case to $\mathscr{N}(R, \rho)$ where $\rho$ is the congruence determined by $\phi$.

Next, let us recall two other constructions which, although they are not extensions, in a sense parallel the construction of $\mathscr{N}(R, \rho)$. Given a standard or positive thread $R$, let $P$ be its order dual and let $x^{\prime}$ be the element of $P$ corresponding to $x$ in $R$. Let $S=P \cup R$ except that we identify $0^{\prime}$ with 0 , and give $S$ the obvious order. Then $\mathscr{R}(R)$ is the thread obtained by defining on $S$ by:

$$
x \circ y=x^{\prime} \circ y^{\prime}=x y, \quad x^{\prime} \circ y=x \circ y^{\prime}=(x y)^{\prime} .
$$


Again, $\mathscr{J}(R)$ is the thread obtained by defining $\circ$ on $S$ by:

$$
x \circ y=x^{\prime} \circ y=x y, \quad x \circ y^{\prime}=x^{\prime} \circ y^{\prime}=(x y)^{\prime} .
$$

It is easy to verify that $\mathscr{R}(R)$ and $\mathscr{J}(R)$ are globally idempotent threads with zero. The construction yielding $\mathscr{R}(R)$ was given in $\S 1$ of [6], while $\mathscr{J}(R)$ is the January thread associated with $R$ defined in the introduction of [1].

1.5 Lemma. Let $S$ be a globally idempotent thread in which $G$ is a positive thread and in which $G L \subset L$. Let $u$ be the identity of $G$ and let $f$ be the unique element in the boundary of $H(u)$. Thus $H(u)=\{s \mid f<s\}$.

(1) If $l<0$, then either $H(u) l=f l$, or right multiplication by $l$ is one-one on $H(u)$. In the latter case, $L$ is unbounded and $G l=L$.

(2) If $L^{2} \leq f$, then $S$ is isomorphic with $\mathscr{R}(G)$.

(3) If $L G \$ f$, then $S$ is isomorphic with $\mathscr{J}(G)$.

Proof. Assume that $s l=t l$ with $f<s<t$. If $r=s t^{-1}$, then $r<u$ so that $r^{n} \rightarrow f$. Thus $t l=s l=r(t l)$ implies that $t l=r^{n}(t l) \rightarrow f(t l)=f l$; and $H(u) l=(H(u) t) l$ $=H(u) f l=f l$.

If $G l \neq L$ or if $L$ is bounded, then there exists an $m$ in $L$ such that $m \leqq G l$. For each $t$ larger than $f, m \leqq t^{-1} l$, and by monotony $t m \leqq t t^{-1} l=u l$. By continuity then, $f m \leqq u l \leqq 0$, and thus $u l=f(u l)=f l$. From this it is obvious that right multiplication by $l$ is not one-one on $H(u)$.

Now let $f<l m$ with $l$ and $m$ in $L$. Then $G=G(l m)=(G l) m \subset(G L) L \subset L^{2}$ so that $u=p q$ with $p$ and $q$ in $L$. Assuming $p \leqq q, q^{2} \leqq p q \leqq p^{2}$; and since the square function is continuous, there exists a $z$ in $[p, q]$ for which $z^{2}=u$. Since $S$ is globally idempotent while $L^{2}=G^{2}=G$, either $G L=L$ or $L G=L$. But $G L=L^{3}=L G$, and it follows that $u$ is an identity on $S$. Thus $z$ belongs to $H(u)$, and by 2.3 and 2.2 of [6], $S$ is isomorphic with $\mathscr{R}(G)$.

To prove (3), let $f<m s$ with $m \leqq 0 \leqq s$. Then $G=G m s \subset L s \subset G$. Thus $s \in H(u)$ and $G=G s^{-1}=L s s^{-1}=L u$; for otherwise, $G=L s=L(s f)=G f$-a contradiction. Let $l$ in $L$ be such that $u=l u$, and define $\theta: G \rightarrow L$ by $\theta(t)=t l$. If $\theta(s)=\theta(t)$, then $s=s(l u)=\theta(s) u=\theta(t) u=t(l u)=t$. Hence $\theta$ is one-one, and by (1), $\theta$ maps $G$ onto $L$. Again, $\theta(s) \theta(t)=(s l)(t l)=s l(u t) l=s(l u) t l=s t l=\theta(s t)$, and $\theta$ is an order reversing isomorphism of $G$ onto $L$. Since $\theta(t) s=(t l)(u s)=t s$ and $s \theta(t)=s(t l)=\theta(s t), S$ is isomorphic with $\mathscr{J}(G)$.

2. Adjunction of endpoints. The first theorem of this section gives sufficient conditions under which a minimal element can be adjoined to a thread in which $L$ is unbounded. Of course, a dual result concerning maximal elements can be proved, and, although we do not state it, we shall occasionally apply it. Theorem 2.2 deals with the case in which a minimal element cannot be adjoined.

2.1 TheOREM. Let $S$ be a globally idempotent thread with a zero in which $L$ is unbounded while $L^{2}$ is bounded from above. If $L s=L$ implies that $s$ is a right identity 
on $L$, and if $s L=L$ implies that $s$ is a left identity on $L$, then the multiplication on $S$ can be extended to the set $T$ obtained by adjoining a minimal element to $S$ in such a way that $T$ becomes a thread.

Before we give the proof, let us point out that the extended thread need not be globally idempotent even though $S$ is. For example, if $S$ is the open interval of real numbers between $-\frac{1}{2}$ and 1 under ordinary multiplication, then $S$ is a thread satisfying the hypotheses of the theorem. However, the extended thread is the interval $\left[-\frac{1}{2}, 1\right)$, and $-\frac{1}{2}$ is not the product of any two elements in this thread.

Proof. Observe that $L$ cannot be the order dual of a positive thread, for in that case there would exist an $l$ in $L$ such that $L l=L$ which is not a right identity on $L$. Thus if $L=S$, then $L^{2}=L$ and zero is a maximal element of $L$, so that $L$ is the order dual of a standard thread without its identity. Since the extension is trivial in this case, we assume that zero is a cut point of $S$.

Suppose that $L x$ is unbounded from above for some $x$. Since zero does not cut $L x, L x=G$ and $G$ is unbounded; and since $L^{2}$ is bounded from above, $x$ is in $G$. Thus $L G=G$ and $L^{2} x=L G=G$. Now if $L^{2} \subset G$ then $L^{2}$ is bounded, and it follows from $L^{2} x=G$ that $G$ is bounded. Thus $L^{2} \subset L$, and as we have seen, $L$ is not the order dual of a positive thread. Hence [6, 5.1 and 3.3], each element of $L$ is $\Gamma$ compact (i.e., the closure of the set of powers of each element in $L$ is compact). Choosing $y$ larger than $x, L x=G$ implies $[0, y] \subset l[0, x]$ for some $l$ in $L$. But this contradicts Theorem 1 in [7] which states that: in any topological semigroup, if $A$ is compact, if $t$ is $\Gamma$-compact, and if $A \subset t A$, then $A=t A$. Hence $L x$ is bounded from above for each $x$ in $S$, and similarly each $x L$ is bounded from above.

Now let $p$ be the minimal element of $T$ and, for $x$ and $y$ in $S$, let

$$
\begin{aligned}
x \circ y & =x y ; & & \\
p \circ x & =\sup L x, & & \text { if } L x \subset G, \\
& =\inf L x, & & \text { if } L x \subset L ; \\
x \circ p & =\sup x L, & & \text { if } x L \subset G, \\
& =\inf x L, & & \text { if } x L \subset L ; \\
p \circ p & =\sup L^{2}, & & \text { if } L^{2} \subset G, \\
& =\inf L^{2}, & & \text { if } L^{2} \subset L .
\end{aligned}
$$

Clearly we are allowing $p$ as the infimum of a set in $S$.

Notice that if $x \prec y$ then $l x \preceq l y$ for $l$ in $L$. That is, $l x \in L y$ for each $l$ so that $L x \subset L y$. Similarly, $x L \subset y L$, and it follows easily that $\circ$ is monotone on $T$ with respect to $\prec$. The next step is to show that $\circ$ is continuous; unfortunately, the proof of this fact is long and involved. However, once this is done the associativity of $。$ follows easily. For $\circ$ extends the given multiplication on $S$ which is associative, and $S$ is a dense subset of $T$.

Letting $S_{0}=\{y \mid y \in S$ and $p \circ y \neq p\}$, we claim that $S_{0}$ is an open connected 
subset of $S$ containing $L$ and that $S_{0}^{2}=S_{0}$. Since this is trivial if $S_{0}=S$, suppose there exists an $x$ such that $p \circ x=p$. Thus $L x=L$, and since $L$ is not the dual of a positive thread, $x$ cannot be in $L$. Each such $x$ is a right identity on $L$, and thus $\{y \mid y \in S$ and $p \circ y=p\}$ is closed both topologically and algebraically. Moreover, if $p \circ y=p$ and if $y<z$, then $p \circ z=p$ by the monotony of $\circ$ on $T$. Thus there is an element $g$ larger than zero such that $S_{0}=\{s \mid s<g\}$. Now $s \in S_{0}$ implies that $I_{s}$ is bounded so that $L s t$ is bounded for any $t$ in $S$. In particular, $S_{0}^{2} \subset S_{0}$. Since $\{y \mid g \leqq y\}$ is also closed algebraically, $g$ is an idempotent. Hence $S_{0}=S_{0} g$ from which it follows that $S_{0} \subset S_{0}^{2}$.

Let $x \in S_{0}$, let $I$ be an open interval in $S$ containing 0 and $p \circ x$, and let $x<w$ with $w$ also in $S_{0}$. Writing $w=u v$ with $u$ in $S_{0}$, let $z$ be the supremum with respect to $\prec$ of $\{t \mid x=u t$ and $t \preceq v\}$. Now $(L u)^{*} z \subset(L u z)^{*}=(L x)^{*}=\{y \mid 0 \preceq y \preceq p \circ x\} \subset I$, and since $u \in S_{0},(L u)^{*}$ is compact. Thus by a familiar result of A. D. Wallace [8, Lemma 2], there is an open set $Z$ in $S$ containing $z$ such that $L u Z \subset I$. Let $z^{\prime}$ in $Z$ be such that $z \prec z^{\prime} \preceq v$ and put $x^{\prime}=u z^{\prime}$. By the definition of $z, x \prec x^{\prime} \preceq w$, and since $p \circ x^{\prime}$ is the supremum of $L u z^{\prime}$ with respect to $\prec, p \circ x^{\prime} \in I^{*}$.

If $x \in S$ and $a \prec p \circ x$, then there exist an $l$ in $L$ and an $x^{\prime \prime}$ in $S$ such that $x^{\prime \prime} \prec x$ and $a \prec l x^{\prime \prime}$. For by the definition of $p \circ x$ there is an $l$ such that $a \prec l x$, and $x^{\prime \prime}$ can then be chosen by continuity.

Now for the actual proof of the continuity of $\circ$, let $x \in S$, assume that $x$ is not a maximal element in $S$, and let $V$ be an open connected set in $T$ containing $p \circ x$. If $p \circ x=p$ or if $p \circ x$ is a maximal element for $S$, let $a \prec p \circ x$ with $a$ in $V$, and choose $l$ and $x^{\prime \prime}$ as above. If $m$ and $q$ are in $T$, if $m<l$, and if $x^{\prime \prime}<q$, then $a<l x^{\prime \prime}=l \circ x^{\prime \prime}$ $\preceq m \circ q$. Thus $\circ$ is continuous at $(p, x)$. Secondly, if $p \circ x=0$ then $x \in S_{0}$, and we choose $w$ and $w^{\prime}$ in $S$ such that $\left(w^{\prime}, w\right)$ contains both 0 and $x$. Let $I$ be an open interval containing 0 such that $I^{*} \subset V \cap S$. Now there are two cases according as $0 \leqq x$ or $x \leqq 0$, and since the two are quite similar, we consider only the first. Then $x<w, p \circ x \in I$, and $x$ and $w$ are both in $S_{0}$, so that there exists an $x^{\prime}$ such that $x \prec x^{\prime} \preceq w$ and $p \circ x^{\prime} \in I^{*}$. Since $p \circ 0 \in I$ and $0 \prec w^{\prime}$, there is likewise an $l$ such that $0 \prec l \preceq w^{\prime}$ and $p \circ l \in I^{*}$. Now $\left(l, x^{\prime}\right)$ is an open set in $T$ containing $x$. If $m$ and $q$ are in $T$, if $m<0$, and if $q \in\left(l, x^{\prime}\right)$, then either $q \prec x^{\prime}$ so that $m \circ q \preceq p \circ x^{\prime}$, or $q \prec l$ and $m \circ q \preceq p \circ l$. In either case $m \circ q \in I^{*} \subset V$, and again $\circ$ is continuous at $(p, x)$. Thirdly, if $p \circ x \neq 0$, if $p \circ x \neq p$, and if $p \circ x$ is not maximal in $S$, then $x \in S_{0}$ and we can choose $w$ in $S_{0}$ such that $x \prec w$. Let $a$ and $b$ in $V \cap S$ be such that $0 \prec a$ $\prec p \circ x \prec b$, and let $c$ be any element in $S$ such that the open interval $I$ between $c$ and $b$ contains zero. On the one hand there are $x^{\prime \prime}$ in $S$ and $l$ in $L$ such that $a<l x^{\prime \prime}$ and $x^{\prime \prime} \prec x$. On the other, there is an $x^{\prime}$ such that $x \prec x^{\prime} \preceq w$ and $p \circ x^{\prime} \in I^{*}$. Now if $m<l$ and if $x^{\prime \prime} \prec q<x^{\prime}$, then $a<l x^{\prime \prime}=l \circ x^{\prime \prime} \preceq m \circ q \preceq p \circ x^{\prime}$. Since $p \circ x^{\prime}$ is in $I^{*}$ and, with respect to $\prec$, is comparable with $a, p \circ x^{\prime} \preceq b$. Thus, $V$ being connected, $m \circ q$ is again in $V$.

Now let $q$ be either $p$ or, in case such exists, the maximal element of $S$, and let $V$ be an open connected set in $T$ containing $p \circ q$. Letting $M$ represent either $L$ or 
$G$ according as $q$ is minimal or maximal, $p \circ q$ is the supremum with respect to $\prec$ of $L M$. If $p \circ q=0$ then $L M=0$ and $\circ$ is clearly continuous at the pair $(p, q)$. If $a \in V$ and $a \prec p \circ q$ then there exist $l$ in $L$ and $m$ in $M$ such that $a \prec l m$. Moreover, employing the continuity of multiplication in $S$, we may assume that $m \prec q$. Since $\circ$ is monotone with respect to $\prec$, it is continuous at $(p, q)$.

We have shown (the hard way) that $\circ$ is continuous at each pair $(p, z)$ with $z$ in $T$. By symmetry, the same is true at each $(z, p)$, and finally, the continuity at $(x, y)$ with both $x$ and $y$ in $S$ is obvious.

2.2 THEOREM. Let $S$ be a globally idempotent thread with zero in which $L$ is not the order dual of a positive thread. If there exists a $z$ which is not a right identity on $L$ yet for which $L z=L$, then $L$ is unbounded, $z \in G$, and $G^{2}=G$. Moreover, if

$$
f=\inf \{x \mid x \in G \text { and } L x=L\}
$$

then

(1) $f$ is the zero of a subthread of $G$ isomorphic with the real interval $[0,1)$ under ordinary multiplication,

(2) $f<t$ implies $L t=L$,

(3) there exists a $p$ in $L$, not minimal, such that $L f=[p, 0]$,

(4) for any $s$, either $s L=L$ or $p \leqq s L$, and

(5) $p \leqq L^{2}$.

Proof. If $l L=L$ or if $L l=L$, then $L^{2}=L$ and $L$ is the order dual of a standard thread or of a standard thread without its identity. But in the latter case, no such $l$ exists, while in the former, the only such $l$ is the identity. In particular, if $L z=L$ and if $z$ is not a right identity on $L$, then $z$ is in $G$.

We know that either $L^{2}=L$ or $G^{2}=G$. If $L^{2}=L$, then, since $L G=L$ as well, either $G^{2}=G$ or $G L=G$. But $G L=G$ implies $G=G L=(G L) L=G(L G) L=(G L)^{2}=G^{2}$. Hence, $G^{2}=G$ in any case.

If $L$ has a minimal element $q$, then by monotony, $q=q z$. But then, each element of $L$ can be written as $q t$ with $t$ in $[0, z]$ and $(q t) z=q(t z)=q(z t)=(q z) t=q t$. Thus $L$ is unbounded, and returning to the argument of the first paragraph, we see that $l L=L$ is impossible for $l$ in $L$.

If $s<t$ and $L s=L$, then by monotony, $L t=L$ as well. From this and the definition of $f$, (2) follows. If $x<f<y$ then $y x \leqq f$; for if not, $L x=(L y) x=L(y x)=L$ and $f \leqq x$. Thus by the continuity and commutativity of multiplication in $G, f y=y f \leqq f$ whenever $f<y$. But $\{y \mid y \in G$ and $L y=L\}$ is obviously a subthread, so that $f$ is an idempotent and a zero for $\{y \mid f \leqq y\}$. If (3) is false than $L f=L$ and $f$, being an idempotent, is a right identity on $L$. This implies that $l z=(l f) z=l(f z)=l f=l$ for each $l$; consequently, (3) is true.

Since $\{y \mid f<y\}$ is the same as $\{y \mid y \in G$ and $L y=L\}$, it is a subthread of $G$. If there were an idempotent in each interval $(f, t)$ with $f<t$, then each of these idempotents would be a right identity on $L$, and by continuity, so would $f$. But $L f \neq L$, 
so there is a $t$ larger than $f$ such that $(f, t)$ contains no idempotents. This establishes (1).

Let $s$ be in $S$ and suppose that $s L \neq L$. If $s L \subset G, p \leqq s L$ is trivial; and if $s L \subset L$ then $s L \subset[l, 0]$ for some $l$. Since $[l, 0]$ is compact, and since $s L=s(L t) \subset[l, 0] t$ for each $t$ larger than $f, s L \subset[l, 0] f \subset[p, 0]$. Hence, either $s L=L$ or $p \leqq s L$. In particular, $p \leqq l L$ for each $l$ in $L$ because $l L=L$ is impossible, and this gives $p \leqq L^{2}$.

3. Normal threads. With any thread various duals are associated: first there is the order dual which is obtained simply by reversing the order; secondly, a new multiplication can be defined by $x \circ y=y x$, while the order is left unchanged, to produce a product dual; finally, both transformations can be performed, yielding a combined order and product dual. Since a description of any one of these four threads can immediately be transferred to the others, we may "normalize" the threads to be studied by placing on them convenient restrictions which, in effect, select one from each such set of four threads. Also, as was shown in the preceding section, endpoints can frequently be adjoined when they are missing in a thread, and certainly a description of the structure of the extended thread is enough to describe the original thread. Thus, it is sufficient to study only those threads to which further endpoints cannot be adjoined. This leads us to say that $a$ thread $S$ is normal if and only if:

(1) $S$ is globally idempotent;

(2) S has a zero and the zero cuts $S$;

(3) $G$ is either a standard thread or a positive thread;

(4) $G L \subset L$, and $G L=0$ implies $L G \subset L$;

(5) either $L$ is compact, or there exists an element s such that $s L=L$, or $L s=L$, but which is not a left, or right, identity on L respectively.

The usefulness of this definition is seen in the following theorem.

3.1 THEOREM. If $S$ is a globally idempotent thread with a cutting zero, then $S$, or one of its duals, is either a normal thread or the result of removing one or both endpoints from a normal thread.

Proof. Let $S$ be a globally idempotent thread having a zero which is a cut point. We show first that, by passing to one of the duals of $S$ if necessary, we can achieve a thread which satisfies (4) and, of course, (1) and (2), and in which $G^{2}=G$.

If $G^{2} \neq G$, then, as indicated in the introduction, $L^{2}=L$. Moreover, $S$ is globally idempotent and thus either $L G=G$ or $G L=G$. Now the order dual or the combined order and product dual of $S$ has the desired properties according as $L G$ or $G L$ is $G$.

If $G^{2}=G$ and $G L \nsubseteq G$, then $S$ itself is the desired thread. If $G^{2}=G$ and $L G \nsubseteq G$, then evidently the product dual of $S$ will suffice. Finally, the only case left is that in which $G^{2}=G$ and $L G \cup G L \subset G$, and with this assumption, the order dual of $S$ again has the desired properties. 
Now suppose that $T$ is a thread satisfying (1), (2) and (4) in which $G^{2}=G$. Then $G$, being itself a globally idempotent thread and having a zero as a least element, must be a positive thread, or a standard thread, or a standard thread without its identity. In the third case, which is the only one in which $T$ does not already satisfy (3), if we consider only $G$ it is clear that the missing identity can be replaced. But we must consider all of $T$, and thus we apply the dual of 2.1. To see that this is legitimate, suppose there is an $s$ which is not a right identity for $G$ yet for which $G s=G$. This, using the dual of 2.2 , implies that $G^{2} \neq G-$ a contradiction. Similarly $s G=G$ implies that $s$ is a left identity, and thus a maximal element can be adjoined to $T$. Although it is not included in the conclusion of 2.1 , the extended thread is still globally idempotent because the new element is obviously an identity on $G$. Evidently, properties (2) and (4) continue to hold.

Finally, assume that $T$ is a thread satisfying all but the last requirement in the definition of normality. Thus $L$ is unbounded, $s L=L$ implies that $s$ is a left identity on $L$, and $L s=L$ implies that $s$ is a right identity on $L$. We must show that $L^{2}$ is bounded from above. But if this is not so, then $G$ is unbounded, and is thus a positive thread, and $L^{2}=G$. Then by $1.5, S$ is isomorphic with $\mathscr{R}(G)$ and there certainly does exist an $s$ in $G$ with $L s=L$ which is not a right identity on $L$. Consequently, a minimal element may be adjoined to $T$ by 2.1. Since the extension clearly satisfies properties (2) through (5), we have only to verify that it is globally idempotent. If, in $T, L^{2}=L$, then $L$ is the order dual of a standard thread without its identity, and the minimal element of the extension has to be an idempotent because of the continuity of multiplication in the extension. If $L^{2} \neq L$ in $T$, then either $L G=L$ or $G L=L$. Assuming the former and letting $u$ be the identity of $G, u$ is a right identity on $T$ and, by continuity, on the extension as well. Similarly, if $G L=L$ in $T$, then $u$ is a left identity on the extension of $T$. Hence, given a globally idempotent thread $S$ with a cutting zero, we have constructed a normal thread by adjoining to $S$, or to one of its duals, at most two endpoints.

We observe that if $R$ is a positive thread or a nonzero standard thread, then both $\mathscr{R}(R)$ and $\mathscr{J}(R)$ are normal threads. Again, if the dual of $P$ is either a positive thread or a nonzero standard thread, then $\mathscr{N}(P, R, \theta)$ is a normal thread for any $\theta$. It follows that $\mathscr{N}(R, \rho)$, is a normal thread whenever $\rho$ is a congruence with closed convex classes on $R$ such that $R / \rho \neq 0$.

3.2 Lemma. Let $T$ be an ideal in a thread $S$ and let $S$ be the contact extension of $T$ by $Q$. If $S$ is normal and if $T$ cuts $S$, then $Q$ is normal. Conversely, if $Q$ satisfies requirements (3) and (5) in the definition of normality, if zero cuts $T$, and if the maximal element of $T$ is a left identity on $T$, then $S$ is normal.

Proof. Let $\phi$ be the natural homomorphism of $S$ onto $Q$, let $e$ be the maximal element of $T$, and, in order to avoid confusing the $L$ 's and $G$ 's, denote those in $Q$ by $L^{\prime}$ and $G^{\prime}$.

Assuming that $S$ is normal and that $T$ cuts $S, Q$ clearly satisfies conditions (1) 
and (2). If $u$ is the identity of $G$, then $\phi(u)$ is an identity for $G^{\prime}$, and $G^{\prime}$ is standard or positive according as $\phi(u)$ is maximal or not [6, Theorem 3.3].

If $L^{\prime} G^{\prime} \phi L^{\prime}$ then there exist an $l$ in $L$ and a $g$ in $G$ such that $e<l g$. Since $(u l) g=u(l g)=l g, u l$ is not in $T$; thus $0 \neq \phi(u) \phi(l)$ and $\phi(u) \phi(l) \in G^{\prime} L^{\prime}$. Also, $G^{\prime} L^{\prime}=\phi(G) \phi(L)=\phi(G L) \subset \phi(L)=L^{\prime}$. This proves condition (4).

If $L$ is compact then $L^{\prime}$, the image of $L$ under a continuous function, is compact. If $s L=L$ then $\phi(s) L^{\prime}=\phi(s L)=L^{\prime}$. Moreover, if $\phi(s)$ is a left identity on $L^{\prime}$, then $s l=l$ whenever $l<T$. Fixing an $l$ less than $T$, each element in $T \cap L$ can be written as $r l$ for some $r$ with $r \prec s$. Now $r$ commutes with $s$; for if they are not both in $G$, then they are both in $L$ and $s L=L$ implies $L^{2}=L$. Thus, $s(r l)=r s l=r l$ so that $s$ is a left identity on all of $L$. Hence, if $s L=L$ but $s$ is not a left identity on $L$, then $\phi(s) L^{\prime}=L^{\prime}$ and $\phi(s)$ is not a left identity on $L^{\prime}$. Since the alternate case where $L s=L$ is, of course, also true, condition (5) is proved.

Turning to the second half of the lemma, zero cuts $S$ because it cuts $T$. Moreover, $0 \neq G L \subset L$ follows immediately from the fact that $e$ is a left identity on $T$. If $G^{\prime}=0$, then $G=[0, e]$ which is a standard thread; otherwise, the unique element of $G$ which maps onto the identity of $G^{\prime}$ is itself an identity on $G$. Thus $G$ is either a standard thread or a positive thread. If $L^{\prime}$ is compact, the least element of $S$ which maps onto the minimal element of $Q$ is a minimal element for $S$. Finally suppose that $L^{\prime} \phi(s)=L^{\prime}$ and that $\phi(s)$ is not a right identity on $L^{\prime}$. Then $L^{\prime} \neq 0$, and if $l<T$ then $\phi(l)=\phi(m) \phi(s)$ for some $m$ in $L$. Since $\phi(m s)=\phi(l) \neq 0, m s=l$. Thus 0 is in $L s$ and $\{l \mid l<T\} \subset L s$, so that $L s=L$. If $s$ were a right identity on $L$, then $\phi(s)$ would clearly be a right identity on $L^{\prime}$.

4. Base threads. Let $S$ be a compact thread with a zero in which $G$ is a unit thread or a nil thread (i.e., in which $G$ is isomorphic either with the real interval $[0,1]$ under ordinary multiplication or with the Rees quotient of $[0,1]$ by the ideal $\left.\left[0, \frac{1}{2}\right]\right)$. Then $S$ is an $\mathscr{R}$-base thread if the maximal element is an identity on $S$ and if $0 \neq L_{2} \subset G$. If the maximal element is an identity and $0 \neq L^{2} \subset L$, then $S$ is an $\mathscr{N}$-base thread. If the maximal element is a left identity on $S$ and if $0 \neq L G \subset G$, then $S$ is a $\mathscr{J}$-base thread. To complete the definitions, a trivial thread consisting of zero alone is also an $\mathscr{R}$-base, an $\mathscr{N}$-base, and a $\mathscr{J}$-base thread.

The $\mathscr{R}$-base and $\mathscr{N}$-base threads were introduced by Cohen and Wade [4]; likewise, the first two parts of the following theorem are simply a restatement of their Theorem 5.6. Looking back at the definitions, it is obvious that the nonzero base threads of each of the three types are normal threads. The zero threads are included for convenience in later results.

Let $U$ be the thread consisting of the real interval $[0,1]$ under the usual multiplication, and consider the threads $\mathscr{R}(U), \mathscr{J}(U)$ and $\mathscr{N}(U, \tau)$ where $\tau$ is the congruence in which each element is congruent only to itself. We may consider each of these threads as being defined on the interval $[-1,1]$, and in each, $[-a, 1]$ represents a subthread whenever $a$ is in $(0,1]$. Putting $K=[-b, c]$ where $c \leqq 1$ and 
$b \leqq a$, it is easy to verify that $K$ is an ideal of the subthread $[-a, 1]$ in $\mathscr{R}(U)$ if and only if $a b \leqq c$ and $a c \leqq b$; in $\mathscr{N}(U, \tau)$ if and only if $a c \leqq b$; and in $\mathscr{J}(U)$ if and only if $a c \leqq b \leqq c$. In this statement the juxtaposition of two elements means, of course, their ordinary real product. Moreover, if $K$ is an ideal, the Rees quotient by $K$ of $[-a, 1]$, considered as a subthread of $\mathscr{R}(U), \mathscr{N}(U, \tau)$ or $\mathscr{J}(U)$, will be a nonzero $\mathscr{R}$-base, $\mathscr{N}$-base or $\mathscr{J}$-base thread provided $-a \circ-a$ is not in $K$ in the first two cases and provided $-a \circ 1$ is not in $K$ in the last. The provisions insure that $0 \neq L^{2}$ and $0 \neq L G$ respectively.

4.1 TheOREM. A thread is a nonzero $\mathscr{R}$-base ( $\mathscr{N}$-base, or $\mathscr{J}$-base) thread if and only if it is isomorphic with the Rees quotient of some subthread $[-a, 1]$ of $\mathscr{R}(U)$, $(\mathscr{N}(U, \tau)$, or $\mathscr{J}(U))$ by a closed convex ideal $K$ which does not contain $-a \circ-a$ $(-a \circ-a$, or $-a \circ 1)$.

Proof. A proof of the first two parts can be found in $\$ 5$ of [4], moreover, the "if" parts were proved in the preceding paragraph. Thus assume that $S$ is a nonzero $\mathscr{J}$-base thread and let $S=[d, e]$.

Let $U$ again be the ordinary real interval $[0,1]$, and let $\phi: U \rightarrow[0, e]$ be either an isomorphism or a homomorphism with kernel $\left[0, \frac{1}{2}\right]$ according as $[0, e]$ is a unit thread or a nil thread. Since $0<d e$, there is an $a$ larger than zero in $U$ such that $\phi(a)=d e$. Let $T$ be the subthread $[-a, 1]$ in $\mathscr{J}(U)$, and denote the product of two elements $x$ and $y$ in $T$ by $x \circ y$. Now define $\psi: T \rightarrow S$ by

$$
\begin{aligned}
\psi(x) & =\phi(x), & & \text { if } 0 \leqq x, \\
& =\phi(-x / a) d, & & \text { if }-a \leqq x \leqq 0 .
\end{aligned}
$$

This function is clearly a continuous, nondecreasing mapping of $T$ onto $S$. Moreover, if $0 \leqq x, y \leqq 1$ and if $0 \leqq s, t \leqq a$, then

$$
\begin{aligned}
\psi(x) \psi(y) & =\phi(x) \phi(y)=\phi(x y)=\psi(x y)=\psi(x \circ y) \\
\psi(x) \psi(-s) & =\phi(x) \phi(s / a) d=\phi(x s / a) d=\psi(-(x s))=\psi(x \circ-s) \\
\psi(-s) \psi(x) & =\phi(s / a) d \phi(x)=(\phi(s / a) e) d \phi(x) \\
& =\phi(s / a) \phi(a) \phi(x)=\phi(s x)=\psi(s x)=\psi(-s \circ x) \\
\psi(-s) \psi(-t) & =\phi(s / a) d \phi(t / a) d=\phi(s / a) \phi(a) \phi(t / a) d \\
& =\phi(s t / a) d=\psi(-(s t))=\psi(-s \circ-t) .
\end{aligned}
$$

Hence $\psi$ is a homomorphism, and, putting $K=\psi^{-1}(0), K$ is clearly a closed convex ideal. In addition, $\psi(-a \circ 1)=\psi(a)=\phi(a)=d e>0$ implies that $-a \circ 1$ is not in $K$. Thus $T / K$ is a nonzero $\mathscr{J}$-base thread.

To see that $\psi$ induces an isomorphism of $T / K$ onto $S$, it will suffice to prove that $\psi$ is one-one except on $K$. If $\psi(-s)=\psi(x)$, then $\psi(-s)=\psi(x)=\psi(0)=0$ because $\psi$ is nondecreasing. If $\psi(x)=\psi(y)$ with $x \neq y$, then $\psi(x)=\psi(y)=0$ by the definition of $\phi$. Finally, suppose that $\psi(-s)=\psi(-t)$ with $s<t$. Then $s / t<1$ so that $\phi(s / t)^{n} \rightarrow 0$ in 
G. Thus, $\psi(-t)=\psi(-s)=\phi(s / a) d=\phi(s / t) \phi(t / a) d=\phi(s / t) \psi(-t)$ implies that $\psi(-t)$ $=\phi(s / t)^{n} \psi(-t) \rightarrow 0 \psi(-t)=0$.

5. Left trivial threads. Imitating the definition of left trivial clans by Cohen and Wade, we say that a thread is left trivial if and only if it is normal, $L^{2}=0$, and $L G \cup G L \subset L$. Our purpose in this section is to characterize all left trivial threads.

Let $R$ be a nonzero standard thread or a positive thread, let $P$ be a nonzero thread whose order dual is either standard or positive, and let $\phi$ and $\psi$ be continuous homomorphisms of $R$ into $P$, each taking zero onto zero, such that the identity of $P$ is in the image of at least one of the two. Indicate the product of two elements $s$ and $t$ in $P$ by $s \cdot t$ and of two elements in $R$ by their juxtaposition.

Let $S=P \cup R \backslash\{0\}$, identify the two zeros, extend the existing orders in $P$ and $R$ by declaring each element of $P$ to be less than each element in $R \backslash\{0\}$, and define a multiplication on $S$ by

$$
\begin{array}{ll}
s \circ t=0, & s \circ x=s \cdot \phi(x), \\
x \circ s=\psi(x) \cdot s, & x \circ y=x y,
\end{array}
$$

where $s$ and $t$ are in $P$ and $x$ and $y$ in $R$. Then $S$ is a left trivial thread (we omit the proof) which we denote by $\mathscr{T}(P, R, \phi, \psi)$.

In case $P$ is the order dual of a positive thread and neither $\phi$ nor $\psi$ is onto, another left trivial thread can be obtained from $S$. Let $e$ be the identity of $P$, let $q$ be such that $H(e)=\{s \mid s<q\}$, and let $a$ be any element in $H(e)$. Now put $z \rho w$ in $S$ if and only if $z=w$ or $a \leqq z, w \leqq q$. If $a \leqq s, t \leqq q$ and if $r \leqq 0$ then $s \circ r=t \circ r=0$. If $0<x$, then either $e \leqq \phi(x) \leqq q$, in which case $s \leqq s \cdot \phi(x) \leqq q$ and $t \leqq t \cdot \phi(x) \leqq q$ so that $a \leqq s \circ x, t \circ x \leqq q$, or $q \leqq \phi(x)$ and $s \circ x=s \cdot \phi(x)=\phi(x)=t \circ x$. Thus $u \rho v$ implies that $u \circ z \rho v \circ z$, and similarly $z \circ u \rho z \circ v$. Thus $\rho$ is a congruence relation on $S$ whose classes are clearly closed and convex. The thread $S / \rho$ is easily seen to be left trivial, and we shall denote it by $\mathscr{T}_{a}(P, R, \phi, \psi)$.

5.1 TheOREM. If $S$ is a left trivial thread, then there exists a thread $P$, which is the order dual of a nonzero standard thread or of a positive thread, and there exist homomorphisms $\phi$ and $\psi$ from $G$ into $P$ such that $S$ is isomorphic with $\mathscr{T}(P, G, \phi, \psi)$ or perhaps with some $\mathscr{T}_{a}(P, G, \phi, \psi)$.

Proof. Define $\sim$ on $G$ by: $x \sim y$ if and only if $l x=l y$ for each $l$ in $L$. This evidently defines a congruence on $G$, and it follows from the monotony and continuity of multiplication in $S$ that the congruence classes are closed and convex. Thus, by $1.4, G / \sim$ is either a standard or a positive thread. Defining $\approx$ on $G$ by: $x \approx y$ if and only if $x l=y l$ for each $l$ in $L, G / \approx$ is also either a standard thread or a positive thread. We will consider three cases according as: first, $L$ is compact; second, either $G / \sim$ or $G / \approx$ is a positive thread; and third, $L$ is not compact and $G / \sim$ and $G / \approx$ are both standard threads. In the first case, it will develop that $P$ is the dual of standard thread; in the other two, of a positive thread. In the first two cases, one of $\phi$ and $\psi$ will be onto; in the third, neither. 
If $L$ is compact, let $m$ be the least element. Since $L^{2}=0$ while $S$ is normal; either $L G=L$ or $G L=L$; and without much loss in generality, we will assume that $L G=L$. Thus the identity, $u$, of $G$ is a right identity on $L, m u=m$, and $m G=L$.

In the second case, we will assume that $G / \sim$ is a positive thread. In particular, $G$ itself must be a positive thread and we may choose $f$ so that $H(u)=\{x \mid f<x\}$. Now $f \sim u$ is impossible because $G / \sim$ is a positive thread, and there exists an $m$ in $L$ such that $m f \neq m u$. By part (1) of $1.5, L$ is unbounded and $m G=L$. Since, in each of the first two cases, there exists an $m$ in $L$ such that $m G=L$, we will combine the two from this point on.

Let $P$ be the order dual of $G / \sim$ and let $\phi$ be the natural homomorphism from $G$ onto $P$. Although $\psi$ is not yet defined, we may consider the underlying space $T$ of $\mathscr{T}(P, G, \phi, \psi)$, and we define $j: T \rightarrow S$ by:

$$
\begin{aligned}
j(t) & =t, & & \text { if } t \in G, \\
& =m \phi^{-1}(t), & & \text { if } t \in P .
\end{aligned}
$$

Observe that $j$ is well defined; for if $x$ and $y$ are in $\phi^{-1}(t)$, then $x \sim y$ so that $m x=m y$. Since $m G=L, j$ maps $T$ onto $S$, and $j$ is clearly nondecreasing.

We claim that $j$ is also one-one. To see this, it is sufficient to consider $j(s)=j(t)$ for $s$ and $t$ in $P$. Choosing $x$ in $\phi^{-1}(s)$ and $y$ in $\phi^{-1}(t)$, we have $m x=m y$; and since $m G=L$, we can write each element in $L$ as $m r$ for some $r$ in $G$. Now $(m r) x=m x r=m y r=(m r) y$, and thus $x \sim y$ and $s=t$. Since $j$ is continuous, one-one and onto, it is a homeomorphism.

Defining $\psi: G \rightarrow P$ by $\psi(x)=j^{-1}(x m), \psi$ is continuous and $\psi(0)=0$. If $x$ and $y$ are in $G$, let $\psi(x)=\phi(z)$ and $\psi(y)=\phi(w)$ (this is possible because $\phi$ maps $G$ onto $P$ ). Then $x m=j \psi(x)=j \phi(z)=m z$, and $y m=m w$ so that

$$
\begin{aligned}
j \psi(x y) & =x y m=x m w=m z w=j \phi(z w)=j(\phi(z) \phi(w)) \\
& =j(\psi(x) \psi(y)) .
\end{aligned}
$$

Since $j$ is one-one, $\psi(x y)=\psi(x) \psi(y)$ and $\psi$ is a homomorphism.

Finally, to verify the homomorphic property of $j$, let $x$ and $y$ be in $G$, let $s$ and $t$ be in $P$, and let $t=\phi(z)$. Then:

$$
\begin{aligned}
j(x \circ y) & =j(x y)=x y=j(x) j(y) ; \\
j(s \circ t) & =j(0)=0=j(s) j(t) \\
j(t \circ x) & =j(t \cdot \phi(x))=j(\phi(z) \cdot \phi(x))=j \phi(z x) \\
& =m(z x)=(m z) x=j(t) j(x) ; \\
j(x \circ t) & =j(\psi(x) \cdot \phi(z))=j(\psi(x) \circ z) \\
& =j \psi(x) j(z) \quad(\text { using the previous line) } \\
& =(x m) z=x(m z)=j(x) j(t) .
\end{aligned}
$$

Thus $S$ is isomorphic with $\mathscr{T}(P, G, \phi, \psi)$. 
Now turn to the third case in which $L$ is unbounded while both $G / \sim$ and $G / \approx$ are standard threads. Notice that this does not preclude the possibility that $G$ is a positive thread; it implies only that, in that case, all the elements of $H(u)$ lie in the same congruence class with respect to both $\sim$ and $\approx$. In particular, whether $G$ is positive or not, each element of $G$ is congruent under both relations to some element of $G$ which is $\Gamma$-compact. Thus $l \leqq 0 \leqq x$ implies that $l \leqq l x$ and $l \leqq x l$.

Since $S$ is a normal thread and $L$ is unbounded, there is an $s$ such that $s L=L$ or $L s=L$ which is not a left or right identity respectively on $L$. The two cases being product duals of each other, we will assume that $s$ is not a right identity on $L$ while $L s=L$. Now by 2.2 , there is a unit thread $[f, g]$ in $G$ and there is an element $p$ in $L$ such that $L f=[p, 0]$ while $L t=L$ whenever $f<t$. Moreover, for any $y$ in $S$, either $p \leqq y L$ or $y L=L$. Observe that $L g=L$ and $g^{2}=g$ imply that $g$ is a right identity on $L$.

We prove next that:

if $l, m \in L$ and if $f \leqq x, y \leqq g$; then $l x=l y<p$ implies $x=y$, and $l x=m x<p$ implies $l=m$.

Suppose that $l x<p$. Then $l \leqq l x<p$ implies $l f \leqq p f=p$ so that $l f=p$. Now if $f \leqq x$ $<y \leqq g$ then $x=y t$ for some $t$ in $[f, g)$. Thus $l x=l y$ implies $l x=l(y t)=(l x) t=(l x) t^{n}$ $\rightarrow(l x) f=p$, and $l x=l y<p$ is impossible. Likewise, if $l<m$ and $l x=m x<p$ then $l g=l<m<p=l f$ so that $m=l t$ with $t$ in $(f, g)$. Thus $l x=m x=(l t) x=(l x) t=(l x) t^{n}$ $\rightarrow(l x) f=p-$ a contradiction.

If $f \leqq x<y \leqq g$, then $x \sim y$ is impossible. For $f<y$ implies that $m y<p$ for some $m$ in $L$, and by (*), $m x=m y$ is impossible. On the other hand, $g$ is a zero for $\{x \mid g \leqq x\}$. Otherwise, $G$ is a positive thread, $g=u$ and $(f, g)$ is contained in a single congruence class. Thus if $g \leqq x$ and $l \leqq 0$, then $l g=l(g x)=(l g) x=l x$; that is, $g \sim x$ whenever $g \leqq x$. Consequently, the class containing $g$ is the maximal element of $G / \sim$, and the image of $[f, g]$ under the natural homomorphism is again isomorphic with the real interval $[0,1]$ under ordinary multiplication. From this it is clear that, by defining the multiplication appropriately, we can extend $G / \sim$ by adjoining the real interval $(1, \infty)$. The result is a positive thread in which $G / \sim$ is the closed interval between the zero and the identity. Take $P$ to be the order dual of this positive thread, and let $\phi$ be the natural homomorphism of $G$ into $P$. If $e$ is the identity of $P$, and if $q$ is so chosen that $H(e)=\{s \mid s<q\}$; then $\phi(G)=[e, 0]$, and $\phi$ restricted to $[f, g]$ is an order reversing isomorphism onto $[e, q]$.

We claim next that if $m \leqq p$, if $x$ and $y$ are in $G$ and if $x m=m y \neq p$; then $x l=l y$ for each $l$ in $L$. If $m \leqq l$ then $l=m t$ for $t$ in $G$, so that $x l=x m t=m y t=m t y=l y$. If $l<m$, then $m=l t$ for $t$ in $[f, g)$, and $(x l) t=x m=m y=l t y=(l y) t$. If $x m<p$, then by (*), $x l=l y$. On the other hand, if $p<x m$ then $p<x l$ as well; for if $x l \leqq p$ then, by monotony, $x m=x l t \leqq p t \leqq p f=p$. Similarly $y<f$, because $f \leqq y$ and $m \leqq p$ imply $x m=m y \leqq p f=p$. Hence $x l=(x l) f=x p=x m f=x m$ and $l y=l(f y)=(l f) y=m y$. The claim is established. 
With this we can now define the homomorphism $\psi$ from $G$ into $P$. Let $x \in G$ and suppose there is an $m$ such that $m \leqq p$ and $x m \neq p$. Since $m g=m \leqq x m$, there is an $x^{\prime}$ in $G$ such that $x m=m x^{\prime}$. And this, as we have seen, implies $x l=l x^{\prime}$ for each $l$ in $L$. If there is no such $m$, then $x l=p=l f$ whenever $l \leqq p$. Again, $p<l$ implies $l=p t$ which in turn gives $x l=(x p) t=p t=l=l f$. Thus for each $x$ in $G$ there is an $x^{\prime}$ such that $x l=l x^{\prime}$ for all $l$. Putting $\psi(x)=\phi\left(x^{\prime}\right), \psi$ is a function from $G$ into $P$ taking zero onto zero.

If $b<\psi(x)=\phi\left(x^{\prime}\right)<a$, choose $w$ in $G$ such that $\phi\left(x^{\prime}\right)<\phi(w)<a$ and such that $\phi(w) \neq \phi(f)$. Since $l w=l f$ for $l \leqq p$ implies $l w=l f$ for all $l$, there is an $m$ such that $m \leqq p$ and $m w \neq p$. Clearly $w<x^{\prime}$ and this $x m=m x^{\prime} \leqq m w$. From this it follows that there exists a $y$ in $[0, x]$ such that $y m=m w \neq p$, which means that $\psi(y)=\phi(w)$. Since $\phi(w) \neq \phi\left(x^{\prime}\right), y<x$; and since $\psi$ is obviously nondecreasing, $y<v<x$ implies that $\psi(x) \leqq \psi(v) \leqq \psi(y)=\phi(w)<a$. If it happens that $b<\psi(G)$, this proves that $\psi$ is continuous at $x$. Otherwise there is a $z$ in $G$ such that $\psi(z)=\phi\left(z^{\prime}\right) \leqq b$. As before, choose $s$ in $G$ so that $\phi\left(z^{\prime}\right) \leqq b<\phi(s)<\phi\left(x^{\prime}\right)$ and so that $\phi(s) \neq \phi(f)$. Again, there is a $t$ in $[0, z]$ such that $\psi(t)=\phi(s)$. It follows that $x<t$ and that $b<\psi(t) \leqq \psi(v) \leqq \psi(x)$ whenever $x<v<t$. Hence $\psi$ is continuous.

The homomorphic property of $\psi$ is easy to verify. For if $\psi(x)=\phi\left(x^{\prime}\right)$ and $\psi(y)=\phi\left(y^{\prime}\right)$, then $x y l=x l y^{\prime}=l x^{\prime} y^{\prime}$ for each $l$ in $L$. Thus $\psi(x y)=\phi\left(x^{\prime} y^{\prime}\right)=\phi\left(x^{\prime}\right) \phi\left(y^{\prime}\right)$ $=\psi(x) \psi(y)$. Hence $\phi$ and $\psi$ are continuous homomorphisms of $G$ into $P$ taking zero onto zero, and $T=\mathscr{T}(P, G, \phi, \psi)$ can be defined. We notice also that $\psi(G) \subset \phi(G)=[e, 0]$, that is, neither $\phi$ nor $\psi$ is onto.

Now fix an element $m$ in $S$ such that $m<p$. If $f<t \leqq g$ then $m \in L=L t$; thus there is an element $l$ in $L$ which, by (*), is unique such that $l t=m$. Let $l=\alpha(t)$. If $f<s<t \leqq g$ and if $\alpha(t) \leqq \alpha(s)$, then by monotony, $m=\alpha(t) t \leqq \alpha(s) t \leqq \alpha(s) s=m$, contrary to (*). Since $m g=m$ implies $\alpha(g)=m$, we see that $\alpha$ is a strictly increasing function from $(f, g]$ into $\{l \mid l \leqq m\}$. If $l \leqq m$, then $m \in[l, p) \subset l(f, g]$ implies $l=\alpha(t)$ for some $t$ in $(f, g]$. Hence, $\alpha$ is a homeomorphism of $(f, g]$ onto $\{l \mid l \leqq m\}$ such that $\alpha(t) t=m$.

Define $j: T \rightarrow S$ by

$$
\begin{aligned}
j(t) & =t, & & \text { if } t \in G, \\
& =m \phi^{-1}(t), & & \text { if } e \leqq t \leqq 0, \\
& =\alpha \phi^{-1}\left(t^{-1}\right), & & \text { if } t \leqq e,
\end{aligned}
$$

where $t^{-1}$ is, of course, the inverse of $t$ in $H(e)$. It is evident that $j$ maps $\{t \mid t \leqq e\}$ homeomorphically onto $\{l \mid l \leqq m\}$, and that $j$ is a nondecreasing mapping of $[e, 0]$ onto $[m, 0]$. It follows that $j$ is a continuous nondecreasing function from $T$ onto $S$ which is one-one except possibly in $[e, 0]$.

Suppose that $0 \leqq x<y \leqq f$ and $m x=m y$. If $l \leqq 0$ then $l f=m t$ for some $t$ in $[0, f]$. Hence, $l x=l(f x)=m t x=m x t=m y t=m t y=l f y=l y$, and $\phi(x)=\phi(y)$. If $f \leqq x<y \leqq g$ and $m x=m y$, then by $(*) m x=m y=p$. Thus, if $s$ and $t$ are in $P$, if $s<t$ and if $j(s)=j(t)$, then $e<s<t \leqq q$ and $j(s)=j(t)=j(q)=p$. In other words, either $j$ is one-one 
and is thus a homeomorphism, or there is an $a$ in $H(e)$ such that $j$ is one-one except on $[a, q]$ and such that $j([a, q])=j(q)=p$.

To verify that $j$ is a homomorphism, we will consider only the nontrivial case where one element $x$ is in $G$ and the other $t$ is in $P$. The product of two elements in $P$ is indicated by $s \cdot t$ and of two elements in $T$ by $z \circ w$.

If $e \leqq t \cdot \phi(x)$, let $\phi(z)=t \cdot \phi(x)$ so that $j(t \circ x)=m z$. Now if $e \leqq t$ and $\phi(y)=t$, then $\phi(y x)=t \cdot \phi(x)=\phi(z)$ implies that $m z=m y x=j(t) j(x)$. And if $t \leqq e$ and $\phi(y)=t^{-1}$, then $\phi(y z)=t^{-1} \cdot(t \cdot \phi(x))=\phi(x)$ implies $m z=\alpha(y) y z=\alpha(y) x=j(t) j(x)$.

If $t \cdot \phi(x) \leqq e$, let $\phi(z)=(t \cdot \phi(x))^{-1}$ so that $j(t \circ x)=j(t \cdot \phi(x))=\alpha(z)$. Now if $e \leqq t$ and $\phi(y)=t$, then $\phi(x y z)=e=\phi(g)$ implies $j(t) j(x) z=m y x z=m g=m$. And if $t \leqq e$ and $\phi(y)=t^{-1}$, then $\phi(x z)=\phi(y)$ implies $j(t) j(x) z=\alpha(y) x z=\alpha(y) y=m$. Either way, $j(t \circ x)=\alpha(z)=j(t) j(x)$.

Thus if $x$ is in $G$ and if $t$ is in $P$ then $j(t \circ x)=j(t) j(x)$. Letting $x^{\prime}$ be such that $\psi(x)=\phi\left(x^{\prime}\right)$ we have also:

$$
\begin{aligned}
j(x \circ t) & =j(\psi(x) \cdot t)=j(t \cdot \psi(x))=j\left(t \cdot \phi\left(x^{\prime}\right)\right) \\
& =j\left(t \circ x^{\prime}\right)=j(t) j\left(x^{\prime}\right)=j(t) x^{\prime}=x j(t)=j(x) j(t) .
\end{aligned}
$$

Hence, $j$ is a homomorphism of $T$ onto $S$. If $j$ is one-one, then $S$ is isomorphic with $\mathscr{T}(P, G, \phi, \psi)$. If $j$ is not one-one and if $a$ is the element in $H(e)$ which was located above, then $j$ induces an isomorphism between $\mathscr{T}_{a}(P, G, \phi, \psi)$ and $S$.

6. Classification. Finally, we are ready to classify all the normal threads; as was shown in 3.1 , this will essentially describe all globally idempotent threads with a cutting zero. First, seven classes of threads will be defined, and then it will be shown that every normal thread falls in one of the seven.

Let $R$ be a standard thread and let $\mathscr{R}(R)=[d, e]$. Since $e$ is an identity on $\mathscr{R}(R)$ and $d^{2}=e$, the contact extension of $\mathscr{R}(R)$ by any $\mathscr{R}$-base thread exists by 1.1 . Let $E$ be any süch extension in which $R$ and the $\mathscr{R}$-base thread are not both zero, so that $0 \neq L^{2} \subset G$ in $E$. Since the maximal element of $E$ is an identity for $E$, the contact extension of $E$ by any left trivial or any standard or positive thread exists, again by 1.1. Let $\mathscr{C}_{1}$ consist of all contact extensions of such $E$ by left trivial threads. Let $\mathscr{C}_{2}$ consist of all contact extensions of such $E$ by standard and positive threads along with all threads isomorphic with $\mathscr{R}(P)$ for some positive thread $P$. It follows easily from 3.2 that all the threads in $\mathscr{C}_{1}$ or in $\mathscr{C}_{2}$ are normal, and it is clear that, in each, $L G \subset L$ and $0 \neq L^{2} \subset G$. If $S$ is in $\mathscr{C}_{1}$, then there exists an $l$, not minimal, such that $m<l$ implies $m^{2}=l^{2}$, while if $S$ is in $\mathscr{C}_{2}$, there is no such $l$.

Let $\mathscr{N}(R, \rho)=[d, e]$ where $R$ is a standard thread and $\rho$ is a congruence relation on $R$ with closed convex classes. Since $e$ is an identity and since $d^{2}=d$, the contact extension of $[d, e]$ by any $\mathscr{N}$-base thread exists by 1.1 . Let $F$ be any such extension for which $R / \rho$ and the $\mathscr{N}$-base thread are not both zero, so that $0 \neq L^{2} \subset L$ in $F$. Since the maximal element of $F$ is an identity on $F$, the contact extension of $F$ by any left trivial or standard or positive thread exists. Let $\mathscr{C}_{3}$ consist of all contact 
extensions of such $F$ by left trivial threads. Let $\mathscr{C}_{4}$ consist of all contact extensions of such $F$ by standard and positive threads along with all threads isomorphic with linear extensions $\mathscr{N}(Q, Z, \theta)$ where $Z$ is a positive thread or a nonzero standard thread, where the order dual of $Q$ is a positive or a nonzero standard thread, and where $\theta$ is a continuous homomorphism of $Z$ into $Q$ such that $\theta(0)=0$. Again by 3.2 and the discussion preceding 3.2, each thread in $\mathscr{C}_{3} \cup \mathscr{C}_{4}$ is normal. Moreover it is clear that each one satisfies $L G \subset L$ and $0 \neq L^{2} \subset L$. If $S$ is in $\mathscr{C}_{3}$, there is an $l$, not minimal, such that $m^{2}=l^{2}$ whenever $m<l$, while if $S$ is in $\mathscr{C}_{4}$, there does not exist such an $l$.

If $R$ is a standard thread then, by 1.1 , the contact extension of $\mathscr{J}(R)$ by any $\mathscr{J}$-base thread exists. Let $H$ be any such extension in which $R$ and the $\mathscr{J}$-base thread are not both zero, so that $0 \neq L G \subset G$ in $H$. Since the maximal element of $H$ is a left identity for $H$, the contact extension of $H$ by any positive or standard thread exists. Moreover, if $M$ is a left trivial thread in which $L G=0$ (i.e., if $M=\mathscr{T}(P, T, \phi, \psi)$ or $M=\mathscr{T}_{a}(P, T, \phi, \psi)$ where $\phi$ is identically zero), then the extension of $H$ by $M$ also exists. Let $\mathscr{C}_{5}$ consist of the contact extensions of such $H$ by all left trivial threads in which $L G=0$. Let $\mathscr{C}_{6}$ consist of all extensions of such $H$ by positive and standard threads along with all threads isomorphic with $\mathscr{J}(P)$ for some positive thread $P$, and all contact extensions of $\mathscr{J}(R)$ by trivial linear extensions $\mathscr{N}(Q, Z, 0)$ where $R$ is a nonzero standard thread, and where $Z$ and the dual of $Q$ are standard or positive threads. All the threads in these two classes are again normal threads, and each satisfies $0 \neq L G \subset G$. If $S$ is in $\mathscr{C}_{5}$, and if $u$ is the identity of $G$ in $S$; then there exists an $l$, not minimal, such that $m<l$ implies $m^{2}=l^{2}$ and $m u=l u$. If $S$ is in $\mathscr{C}_{6}$ then such an $l$ does not exist.

Let $\mathscr{C}_{7}$ be the class of left trivial threads. By definition, each left trivial thread is normal and each satisfies $L G \subset L$ and $L^{2}=0$.

The remarks made immediately after each definition prove that these seven classes are mutually disjoint. Moreover, the following theorems will demonstrate that these remarks actually characterize the various classes. Thus, since any normal thread obviously satisfies one of the sets of remarks, the promised description of all normal threads lies in the construction of these classes.

6.1 LeMMA. Let $S$ be a compact normal thread, and let $S=[p, e]$.

(1) If $e$ is a right identity on $S$, if $0 \leqq r<e$ implies that $p r \neq p$, and if $p<l \leqq 0$ implies that $p^{2} \neq l^{2}$; then $S$ is one of the threads E occurring in the definitions of $\mathscr{C}_{1}$ and $\mathscr{C}_{2}$, or one of the threads $F$ occurring in those of $\mathscr{C}_{3}$ and $\mathscr{C}_{4}$, according as $0<p^{2}$ or $p^{2}<0$.

(2) If $e$ is a left identity on $S$, and if $e$ is the only idempotent in [pe, e]; then $S$ is one of the threads $H$ occurring in the definitions of $\mathscr{C}_{5}$ and $\mathscr{C}_{6}$.

Proof. We claim first that if $S$ satisfies the hypotheses of (1), then $e$ is in fact an identity on $S$. For $p^{2}=(p e) p=p(e p)$, and since $S$ is normal, $e p \leqq 0$. By 5.1 in [6] then, $e p \leqq p^{2}$ so that $p^{2} \in e S$. Hence $p^{2}=e p^{2}=e(p e p)=(e p)^{2}$, and by hypothesis, $p=e p$. Consequently, $S$ is a pointed clan in the terminology of Cohen and Wade 
and, as such, is commutative. Part (1) now follows from a careful reading of 6.2 and 6.3 in $[4]\left({ }^{3}\right)$.

Now let $S$ satisfy the hypotheses of part (2). Since $e$ is the only idempotent in [pe,e], $0<p e$. Moreover, if $g$ is the largest idempotent in $[0, p e]$, then either $g=p e=e$, or $g<p e<e$ and $[g, e]$ is a unit or nil thread.

Defining $\theta:[0, g] \rightarrow[g p, 0]$ by $\theta(x)=x p, \theta$ is clearly continuous, nonincreasing and onto. If $0 \leqq x, y \leqq g$, then $\theta(x) \theta(y)=(x p)(y p)=x p(e y) p=x(p e) y p=x y p=\theta(x y)$. And if $\theta(x)=\theta(y)$, then $x=x(p e)=(x p) e=(y p) e=y(p e)=y$. Thus $\theta$ is an order reversing isomorphism of $[0, g]$ onto [gp, 0]. Again, $\theta(x) y=(x p)(e y)=x(p e) y=x y$, and $x \theta(y)=x(y p)=(x y) p=\theta(x y)$. It follows from the definition before 1.5 that $[g p, g]$ is isomorphic with $\mathscr{J}([0, g])$.

Since $[0, e]$ is a standard thread and $0<p e, S g=[0, g]$ and $g S=[g p, g]$. Thus $[g p, g]$ is an ideal of $S$, and by $1.2, S$ is the contact extension of $[g p, g]$ by $S /[g p, g]$. If $g=p e=e$ then $S /[g p, g]$ is the zero $\mathscr{J}$-base thread and $[0, g]$ is a nonzero standard thread. If $g<p e<e$, then $S /[g p, g]$ is a compact thread in which $G$ is a unit or nil thread, in which the maximal element is a left identity, and in which $0 \neq L G \subset G$; that is, $S /[g p, g]$ is a nonzero $\mathscr{J}$-base thread.

6.2 Theorem. Let $S$ be a normal thread in which $L G \subset L$ and $L^{2} \neq 0$. Assume that there exists a $q$ in $L$, not minimal, such that $m<q$ implies $m^{2}=q^{2}$. Then if $L^{2} \subset G, S$ is in $\mathscr{C}_{1}$, and if $L^{2} \subset L, S$ is in $\mathscr{C}_{3}$.

Proof. From the hypothesis on $q$, it is clear that $L^{2} \neq L$; therefore, either $L G=L$ or $G L=L$. Since the two cases are similar, we will assume $L G=L$, which implies that $u$, the identity of $G$, is a right identity on $S$.

Put $p=\sup \left\{l \mid l \leqq 0\right.$ and $\left.l^{2}=q^{2}\right\}$ and $e=\inf \{x \mid 0 \leqq x$ and $p=p x\}$. If $l \leqq m \leqq p$, then by monotony, $m^{2} \preceq m l, l m \preceq l^{2}$, so that $l m=m l=p^{2}$. Thus $L^{2}=[p, 0]^{2}$, and in particular, $p^{2} \neq 0$. It is clear that $e$ is an idempotent larger than zero and that $p=p e$. If $e<p^{2}$ then, since $p^{2}=p^{2} e, G$ is a positive thread with $u=e$; and by $1.5, S$ is isomorphic with $\mathscr{R}(G)$, contrary to the hypothesis concerning $q$. Similarly, $p^{2}<p$ implies, by 5.1 in [6], that $L$ is the dual of a positive thread, and this again contradicts the hypothesis on $q$. We know that $p=p e$; and if $e p \leqq p$, then $p \in e S$ so that $p=e p$. Thus $p^{2}, e p$, $p e$ and $e^{2}$ are in $[p, e]$, and $[p, e]$ is, by monotony, a subthread of $S$. This subthread clearly satisfies 6.1 and is thus one of the threads $E$ or $F$ according as $L^{2} \subset G$ or $L^{2} \subset L$ in $S$; in particular, [ $\left.p, e\right]$ is commutative.

Now suppose that $l<p$ and $l e=l$. Then $p=l$ for some $t$ less than $e$, so that $p^{2}=p(l t)=(p l) t=p^{2} t$. It follows that $p^{2}=p^{2} t^{n} \rightarrow p^{2} h$ where $h$ is the largest idempotent in $[0, t]$. Thus $p^{2}=(p h)^{2}$ which implies $p=p h$ which in turn implies $h=e$. But $h \leqq t<e$. Hence $e$ is not $u$, and $S e=[p, e]$. Similarly, $e l=l<p$ implies $p=t l$ for some $t<e$, and thus $p^{2}=t p^{2}=p^{2} t$-a contradiction. Consequently, $[p, e]=e S=S e$, and by $1.2, S$ is the contact extension of $[p, e]$ by $S /[p, e]$.

( $\left.{ }^{3}\right)$ See the referee's comment at the end of the paper. 
Finally, $S /[p, e]$ is a normal thread by 3.2 , and it clearly satisfies $L G \subset L$ and $L^{2}=0$. That is, $S /[p, e]$ is a left trivial thread and $S$ is in $\mathscr{C}_{1}$ or $\mathscr{C}_{3}$ according as $L^{2} \subset G$ or $L^{2} \subset L$.

6.3 TheOREM. Let $S$ be a normal thread in which $L G \subset L$ and $L^{2} \neq 0$. Assume that, for each $l$ which is not minimal, there exists an $m$ such that $m<l$ and $m^{2} \neq l^{2}$. Then if $L^{2} \subset G, S$ is in $\mathscr{C}_{2}$, and if $L^{2} \subset L, S$ is in $\mathscr{C}_{4}$.

Proof. Suppose first that $L$ has a minimal element $p$, and that $L^{2} \neq L$. Then $p<l \leqq 0$ implies $p^{2} \neq l^{2}$; for if $p^{2}=l^{2}$, then by monotony, $m^{2}=l^{2}$ for each $m$ in [ $p, l]$. Since $L^{2} \neq L$, either $L G=L$ or $G L=L$; and as usual, we will treat only one case-say $L G=L$. This means that $p u=p$ where $u$ is the identity of $G$, and putting $e=\inf \{x \mid 0 \leqq x$ and $p x=p\}, e$ is evidently a nonzero idempotent and a right identity on $L$. Moreover, $e$ is a zero for $Z=\{x \mid e \leqq x\}$ and $Z$ is either a standard or a positive thread. This last claim is obvious if $G$ is standard, while if $G$ is a positive thread with $H(u)=\{x \mid f<x\}$, it follows from part (1) of 1.5 that $p f=p u=p$. Since this means that $e \leqq f, Z$ is a positive thread with $e$ as zero. From these facts it follows that $e S \subset[p, e]=S e$, that is, $[p, e]$ is an ideal. Since $[p, e]$ clearly satisfies the hypotheses of part (1) of 6.1 , it is an $E$ or an $F$ according as $0<p^{2}$ or $p^{2}<0$, and in particular, $e$ is an identity on $[p, 0]$. By $1.2, S$ is the contact extension of $[p, e]$ by $S /[p, e]$ which is isomorphic with $Z$. Thus $S$ is in $\mathscr{C}_{2}$ or $\mathscr{C}_{4}$ according as $L^{2} \subset G$ or $L^{2} \subset L$.

Next suppose that $L$ is the order dual either of a standard thread or of a positive thread. In either case $L$ has an identity $e$, and we define $\theta: G \rightarrow L$ by $\theta(x)=e x$. Then $l \leqq 0 \leqq x$ implies $l x=(l e) x=l \theta(x)$ and $x l=e(x l)=\theta(x) l$. Since $\theta$ is clearly a continuous homomorphism of $G$ into $L$ taking zero onto zero, $S$ is isomorphic with $\mathscr{N}(L, G, \theta)$ and so is in $\mathscr{C}_{4}$.

The only case remaining is that in which $L$ is not compact and $L$ is not the dual of a positive thread. We claim that if $h$ is an idempotent in $G$, then $h L=L$ if and only if $L h=L$. For suppose that $h L=L$ while $L h=[m, 0]$ where $m \leqq 0$. If $l<m$ then $l^{2}=l(h l)=(l h) l=m l$. Now if $m l$ were less than $m$ then $L$ would be the dual of a positive thread [6, Lemma 5.1], and thus either $m l \in L h$, in which case $l^{2}=m l=(m l) h=m(l h)=m^{2}$, or $m l \in G$ so that $l^{2}=h(m l)=(m l) h=m^{2}$. Since this contradicts the hypothesis, $h L=L$ implies $L h=L$, and similarly $L h=L$ implies $h L=L$.

Since $S$ is normal and $L$ is not compact, there exists an $s$ such that $s L=L$ and $s$ is not a left identity or such that $L s=L$ and $s$ is not a right identity on $L$, and we will assume the latter. By 2.2, there is a unit thread $[f, g]$ in $G$ and there is an element $p$ in $L$ such that $L f=[p, 0]$ while $L t=L$ whenever $f<t$. In addition, $p \leqq L^{2}$ and, for any $x$, either $p \leqq x L$ or $x L=L$. Since $f<g, L g=L$; and thus $g L=L$. Again, $L f \neq L$ implies $p \leqq f L$. Choosing $l<p, g l=l<p \leqq f l$ implies $t l=p$ with $t$ in $[f, g)$. Consequently, $p=p f=t l f=t p=t^{2} p=t^{n} p \rightarrow f p$. If, by way of contradiction, $L^{2} \leqq f$, then $L^{2} \subset[p, f] \subset S f$. But then $l \leqq p$ implies $l^{2}=l^{2} f=l(l f)=l p=l(f p)=(l f) p=p^{2}$. 
Hence, $L^{2} \leq f$, so that $L^{2} \leq t$ for some $t$ larger than $f$. This means that $L^{2} \subset G$, $L^{2} t=L^{2}$ yet $L^{2} థ[0, t]$. No such $t$ could exist if $G$ were a standard thread, so $G$ is positive, $t \in H(u)$, and by $1.5, S$ is isomorphic with $\mathscr{R}(G)$. This puts $S$ in $\mathscr{C}_{2}$.

6.4 TheOREM. Let $S$ be a normal thread in which $0 \neq L G \subset G$, and let $u$ be the identity of $G$. If there exists a $q$ in $L$, not minimal, such that $m<q$ implies $m^{2}=q^{2}$ and $m u=q u$, then $S$ is in $\mathscr{C}_{5}$.

Proof. Assuming that such a $q$ exists, it is clear that $L^{2} \neq L$; and since $L G \subset G$ while $S$ is globally idempotent, $G L=L$ and $u$ is a left identity on $S$. Letting $p=\sup \{l \mid l \leqq 0$ and $l u=q u\}$, it is evident that $p$ is not minimal, that $l u=p u$ whenever $l \leqq p$, and that $0 \leqq l u<p u$ whenever $p<l \leqq 0$. Moreover, $p u \neq 0$ and $p \neq 0$ follow from $L G \neq 0$.

If $G$ is a positive thread and if $p u \in H(u)$ then, by $1.5, S$ is isomorphic with $\mathscr{J}(G)$, which entails $L^{2}=L$. Thus, whether $G$ is positive or not, $p u \leqq u$; and taking $e$ to be the least idempotent in $[p u, u], e$ is a zero for $\{x \mid e \leqq x\}$. If $e l=l$ for some $l$ less than $p$, then $p=t l$ for some $t$ less than $e$. Letting $h$ be the largest idempotent in $[0, t]$, this gives $p u=t l u=t(p u)=t^{2}(p u)=t^{n}(p u) \rightarrow h(p u)$, and $p u \leqq h<e-$ contrary to the choice of $e$. Thus $e S \subset[p, e]$, and $e<u$. Note also that $S e=[0, e]$.

Now $p u \leqq e$ implies $(e p) u=e(p u)=p u$, and by the definition of $p, e p=p$. Thus $[p, e]$ is $e S$ which is an ideal. Again, $p u=(p u) e=p(u e)=p e$, so that $0<p e$ and $e$ is the only idempotent in [pe, e]. By 6.1 and $1.2,[p, e]$ is one of the threads $H$ occurring in the definition of $\mathscr{C}_{5}$, and $S$ is the contact extension of $[p, e]$ by $S /[p, e]$. By $3.2, S /[p, e]$ is a normal thread, and since $L u=[0, p u] \subset e S, L S=L(u S) \subset e S=[p, e]$. Thus, $S /[p, e]$ is a left trivial thread in which $L G=0$.

6.5 Theorem. Let $S$ be a normal thread in which $0 \neq L G \subset G$, and let $u$ be the identity of $G$. If, for each $l$ in $L$ which is not minimal, there exists an $m$ less than $l$ such that either $m^{2} \neq l^{2}$ or $m u \neq l u$, then $S$ is in $\mathscr{C}_{6}$.

Proof. Suppose that $L$ is not compact and that $L$ is not the order dual of a positive thread. Since $S$ is normal, there exists an $s$ such that $L s=L$, or $s L=L$, which is not a right, or left, identity on $L$. This $s$ must be in $G$, for $L$ is not the dual of a positive thread. Further, $L s=L$ is then impossible because $L G \subset G$. Now by 2.2 , or rather its product dual, there exist a unit thread $[f, g]$ in $G$ and an element $p$ in $L$ such that $f L=[p, 0], t L=L$ for $f<t$, and $p \leqq L^{2}$. Evidently, $u$ is a left identity on $L$, but if $u \in L u$ then $L=u L \subset(L u) L=L(u L)=L^{2}$, which is impossible. Thus, for each $t$ larger than $f, L u=t L u \subset t[0, u)=[0, t)$; consequently, $L u \subset[0, f]$. Now $l<p$ implies that $l u=f(l u)=(f l) u=p u$ and that $l^{2}=l(u l)=(l u) l=(f(l u) f) l$ $=(f l)^{2}=p^{2}$. This contradicts the hypothesis, and thus $L$ is either compact or the order dual of a positive thread.

Assuming now that $L^{2}=L, L$ is either the dual of a standard thread or the dual of a positive thread. In either case $L$ has an identity $e$. Recall from the definition 
of normality that $0 \neq L G \subset G$ implies $0 \neq G L \subset L$. In particular, $u e<0<e u$, and from this we obtain:

$$
\begin{aligned}
& (e u)^{2}=e(u(e u))=e(e u)=e u \\
& (u e)^{2}=u(e(u e))=u(u e)=u e, \\
& (e u)(u e)=e(u e)=u e \\
& (u e)(e u)=u(e u)=e u
\end{aligned}
$$

That is, $[0, e u]$ is a nonzero standard thread and [ue,eu] is a subthread of $S$ isomorphic with $\mathscr{J}([0, e u])$.

If $e u$ does not cut $e u S e u$ and if $u e$ does not cut $u e S u e$, then $Z=\{x \mid e u \leqq x\}$ is a standard or positive thread and $Q=\{l \mid l \leqq u e\}$ is the order dual of a standard or positive thread. Since $G L=G u e L=G(e u)(u e) L=[0, e u][u e, 0]=[u e, 0]$ and since $L G=L e u G=L(u e)(e u) G=[u e, 0][0, e u]=[0, e u] ;[u e, e u]$ is an ideal of $S, S$ is the contact extension of [ue,eu] by $S /[u e, e u]$, and $S /[u e, e u]$ is a globally idempotent thread in which $L G=G L=0$. It is clear that $S /[u e, e u]$ is isomorphic with the trivial linear extension $\mathscr{N}(Q, Z, 0)$, and thus $S$ is in $\mathscr{C}_{6}$.

If $e u$ does cut $e u S e u$, then $G$ is a positive thread with $e u$ as identity [6, Theorem 3.3], and $S$ is isomorphic with $\mathscr{J}(G)$ by 1.5. Similarly, if ue cuts ueSue then $S$ is isomorphic with $\mathscr{J}(G)$ and $G$ is a positive thread. Again, $S$ is in $\mathscr{C}_{6}$.

Finally, assume that $L^{2} \neq L$. Then $L$ must be compact, and we let $p$ be the minimal element. Since $L^{2} \neq L$ and $L G \subset G, u$ is a left identity on $S$.

Notice that $0 \neq L G \subset G$ implies $0<p u$. If $G$ is a positive thread and $p u \in H(u)$, then $S$ is isomorphic with $\mathscr{J}(G)$ by 1.5 , which means that $L^{2}=L$. Thus, whether $G$ is positive or not, $p u \leqq u$, and taking $e$ to be the least idempotent in $[p u, u], e$ is a zero for $Z=\{x \mid e \leqq x\}$ and $Z$ is either a standard or a positive thread.

Observe that $p e=p(u e)=(p u) e=p u$, that $(e p) u=e(p u)=p u$, and that $(e p)^{2}=e(p e) p$ $=e(p u) p=p u p=p^{2}$. It follows that $0<p e$, that $e p=p$, and that $e$ is the only idempotent in $[p e, e]$. Since $e S=[p, e]$ and $S e=[0, e],[p, e]$ is an ideal and $S$ is the contact extension of $[p, e]$ by $S /[p, e]$. Moreover, the quotient thread is obviously isomorphic with $Z$, and $[p, e]$ is one of the threads $H$ occurring in the definitions of $\mathscr{C}_{5}$ and $\mathscr{C}_{6}$. That is, $S$ is in $\mathscr{C}_{6}$.

7. Co s lusion. Let $T$ be a globally idemr tent thread. If $T$ has a zero and if the zero is an endpoint, then, as is stated in the introduction, the structure of $T$ is known. The remainder of this paper has described the structure of $T$ in the event that $T$ has a cutting zero.

If $T$ has no minimal ideal at all, than [6, Theorem 4.1], a zero can be adjoined to $T$ as an endpoint. Again, the structure of $T$ can be determined.

Now suppose that $T$ does have a minimal ideal $K$, but that $K$ is not simply a zero. According to 4.2 of [6], $K$ is then a closed connected set of left zeros, or a closed connected set of right zeros, or $K$ is isomorphic with the group of positive real numbers. In this last possibility, $K$ must be all of $S$, and one could hardly ask 
for a nicer description of $S$. In case $K$ is a proper ideal of $S$, then $S / K$ is again a globally idempotent thread with a zero.

Hence, the only step missing in a description of all globally idempotent threads is a determination of all possible extensions of a thread of one-sided zeros by a globally idempotent thread with a two-sided zero. The special case of this problem in which the threads involved have idempotent endpoints has been solved by Clifford in [2]. Undoubtedly, the general case would be quite similar.

Referee's comments. Theorem 6.2 of Cohen and Wade [4] asserts that if $S$ is a compact thread $[p, e]$ with zero and with identity element $e$, and if $p^{2} \geqq 0$, then $S \in \mathscr{S}(K, k)$ where $K=[0, e]$ and $k=p^{2}$. The class $\mathscr{S}(K, k)$ of threads, described prior to their Theorem 6.2, consists of the following six types.

$S_{1}=\mathscr{R}(K)$ with $K$ a standard thread.

$S_{2}$ is the contact extension of some $S_{1}$ by a nonzero standard thread.

$S_{3}$ is the contact extension of some $S_{1}$ by a left trivial thread.

$S_{4}$ is the contact extension of some $S_{1}$ by an $\mathscr{R}$-base thread.

$S_{5}$ is the contact extension of some $S_{4}$ by a left trivial thread.

$S_{6}$ is the contact extension of some $S_{4}$ by a nonzero standard thread.

If $p^{2}>0$ then our thread [ $p, e$ ] satisfies the hypotheses of Theorem 6.2 of [4], and so must be one of the foregoing types. Types $S_{3}$ and $S_{5}$ are excluded by the hypothesis that $p<1 \leqq 0$ implies $p^{2} \neq l^{2}$. Types $S_{2}$ and $S_{6}$ are excluded by the hypothesis that $0 \leqq r<e$ implies $p r \neq p$. This leaves $S_{1}$ and $S_{4}$, that is, a thread of type $E$ occurring in the definition of $\mathscr{C}_{5}$. Similarly, if $p^{2}<0,[p, e]$ satisfies the hypotheses of Theorem 6.3 of [4], and we see in a similar manner that [p,e] must be a thread of the type $F$ occurring in the definition of $\mathscr{C}_{6}$.

\section{REFERENCES}

1. A. H. Clifford, Connected ordered topological semigroups with idempotent endpoints. I, Trans. Amer. Math. Soc. 88 (1958), 80-98.

2. - Connected ordered topological semigroups with idempotent endpoints. II, Trans. Amer. Math. Soc. 91 (1959), 193-208.

3. - Extensions of semigroups, Trans. Amer. Math. Soc. 68 (1950), 165-173.

4. Haskell Cohen and L. I. Wade, Clans with zero on an interval, Trans. Amer. Math. Soc. 88 (1958), 523-535.

5. P. S. Mostert and A. L. Shields, On the structure of semigroups on a compact manifold with boundary, Ann. of Math. 65 (1957), 117-143.

6. C. R. Storey, The structure of threads, Pacific J. Math. 10 (1960), 1429-1445.

7. A. D. Wallace, Inverses in Euclidean mobs, Math. J. Okayama Univ. 3 (1953), 23-28.

8. ——, A note on mobs, An. Acad. Brasil. Ci. 24 (1952), 329-334.

Princeton University, Princeton, New Jersey 